\title{
A randomized controlled trial to assess the postoperative analgesic efficacy of USG guided adductor canal block compared to conventional analgesia among the patients undergoing arthroscopic anterior cruciate ligament reconstruction
}

\author{
Shruti Ghodageri ${ }^{1 *}$, G. Rammohan ${ }^{2}$, Ashwini Narasannavar ${ }^{3}$, Gajanan Fultambkar ${ }^{4}$, Rashmi B.M. ${ }^{5}$ \\ ${ }^{1,3,5}$ Assistant Professor, ${ }^{2,4}$ Consultant, ${ }^{1,2,4}$ Dept. of Anaesthesia, ${ }^{3}$ Dept. of Public Health, ${ }^{5}$ Dept. of Community Medicine, ${ }^{1,5}$ Basaveshwara \\ Medical College \& Hospital, Chitradurga, Rajiv Gandhi University of Health Sciences, Bangalore, Karnataka, ${ }^{2,4}$ Yashoda Hospital, \\ Secunderabad, ${ }^{3}$ J.N. Medical College, Belagavi Karnataka, India
}

*Corresponding Author: Shruti Ghodageri

Email: shrutib.ghodageri28@gmail.com

Received: $23^{\text {rd }}$ February, 2019

Accepted: $25^{\text {th }}$ March, 2019

\begin{abstract}
Arthroscopic knee surgery can cause significant postoperative pain which has to the potential to delay timely discharge from ambulatory surgical setting. The ultrasound guided adductor canal block may provide superior analgesia over traditional distal saphenous nerve blocks for surgical procedures of knee because ACB block includes saphenous nerve, the nerve to vastus medialis, and potentially the articular contribution of obturator nerve to knee joint. The aim of the study was to compare the postoperative analgesic efficacy of ultrasound guided adductor canal block (ACB) in patients undergoing Arthroscopic Anterior cruciate ligament (ACL) reconstruction with the conventional tramadol analgesia.

Materials and Methods: The prospective randomized controlled study was conducted at a tertiary care hospital in Andhra Pradesh among 60 patients with grade I/ II ASA (American Society of anesthesiologists classification) 1 \& 2, posted for elective Arthroscopic Anterior cruciate ligament reconstruction who were randomly allocated into each of the two groups: conventional analgesia (tramadol) group and ACB group (Adductor canal block with $20 \mathrm{ml}$ of $0.75 \%$ ropivacaine).

Results: NRS static and dynamic Scores among the patients of ACB group were significantly lower than those among the patients of conventional Tramadol group. The first request for analgesia was significantly earlier in conventional tramadol group (1.717 \pm 0.19 hours) when compared with the ACB group ( $15.33 \pm 0.75$ hours).the dose frequency also the total dosage of tramadol among patients in conventional tramadol group (3.60+0.674) was significantly higher than that among the patients in ACB group. Conclusion: this RCT demonstrates that USG guided ACB block, as a component of multimodal analgesic regimen provides superior analgesia when compared to conventional analgesia during the $24 \mathrm{hr}$ postoperative period in patients undergoing elective Arthroscopic ACL repair.
\end{abstract}

Keywords: Adductor canal block, Arthroscopic anterior cruciate ligament reconstruction, Randomized controlled trial, Tramadol, Analgesia, dosage and frequency of tramadol, NRS static and dynamic pain scores.

\section{Introduction}

Anterior cruciate ligament (ACL) injury is one of the most common athletic injuries and is usually treated by arthroscopic ACL reconstruction surgeries. Arthroscopic knee surgery can cause significant postoperative pain which has the potential to delay timely discharge from ambulatory surgical setting. There are various techniques available to achieve postoperative analgesia such as by spinal, epidural anesthesia, local anesthetic wound infiltration, intra-articular injections and peripheral nerve block, traditional intravenous opioid administration (patient control analgesia). ${ }^{1}$

Even though these methods are useful in postoperative pain management, they have their own limitations. Opioids used in PCA are associated with side effects such as postoperative nausea and vomiting (PONV), tiredness, pruritus, constipation and headache. Also, pain relief on movement is not adequately controlled by PCA method even with moderate to large doses of drug. Spinal and epidural anesthesia may lead to hypotension and motor blockade that might cause delay in the patient ambulation and physiotherapy goals..$^{2-4}$ Even though femoral nerve block is better than intra-articular anesthetic injections for less invasive ambulatory knee surgeries, the femoral nerve block usage is limited due to its potential to cause motor blockade of quadriceps which further increases the risk of falling of patients. ${ }^{5-7}$

Adductor canal block has been the new area of research interest to achieve effective postoperative analgesia. The local anesthetics deposited in adductor canal provide improved analgesia by blocking saphenous nerve which is a terminal branch of femoral nerve. The advantage of ACB is that it exclusively blocks sensory innervations, thus reducing pain as well as opioid consumption after knee surgery. ${ }^{8}$ Ultrasound guided adductor canal block is hypothesized to provide superior analgesia over landmark based saphenous nerve block technique which has only $33 \%$ success rates for surgical procedures of knee. ${ }^{9-11}$

With this background, the present study was conducted to evaluate the analgesic efficacy of ultrasound guided adductor canal block as part of multimodal analgesic regimen in order to achieve improved analgesia immediately following arthroscopic procedure. The aim of the study was to compare the postoperative analgesic efficacy of ultrasound guided adductor canal block (ACB) in patients undergoing arthroscopic anterior cruciate ligament (ACL) reconstruction with the conventional tramadol analgesia. The objectives of the study were : a) To estimate the difference in the time of first request of analgesic among the two groups, b) To 
compare the Dosage of opioid (tramadol) requirement during the first 24 hours among the two groups c) To compare the frequency of opioid (tramadol) requirement during the first 24 hours among the two groups and d) To compare the postoperative pain relief in patients of both groups using the Numerical rating scale (NRS) at rest and at movement.

\section{Materials and Methods}

The prospective randomized controlled study was conducted after obtaining institutional ethical clearance, at a tertiary care Yashoda hospital in Secunderabad, Telangana, India, from 1st September 2015 - 30th May 2016 (1year). Study population consisted of a total of 60 patients with ASA (American Society of Anesthesiologists classification) $1 \& 2$, posted for elective Arthroscopic Anterior cruciate ligament reconstruction, who were included in the study after obtaining their informed consent. Sample size was decided in consultation with a statistician and based on pilot study which was carried out in the same hospital. Considering the occurrence of breakthrough pain at $14 \mathrm{hrs}$ in the ACB group to be clinically relevant, with the tramadol group developing pain earlier. Assuming alpha $=0.05$ and power $=0.8$, with mean \pm SD in group 1 (tramadol) $=310 \pm 130$ and group 2 (ACB) mean $\pm \mathrm{SD}=210 \pm 85$ resulted in 21 patients in each group. Sample size calculation: - Sample size was estimated using the formula: $2 \mathrm{SD}^{2}\left(\mathrm{Z}_{\alpha / 2}+\mathrm{Z}_{\beta}\right)^{2} \div \mathrm{d}^{2}$, Where; SD: Standard deviation from pilot study $=\operatorname{Std}$. Dev (130-85) $Z \alpha / 2 ; 1.96$, Z $\beta$ $=0.841$ (from $\mathrm{Z}$ table) at $80 \%$ power. $\mathrm{d}=$ effective size $=$ difference between mean value $=$ Mean (310-210).

A total of thirty patients were randomly allocated into each of the two groups (Group 1: with conventional analgesia group; Group 2: ACB group -Adductor canal block with $20 \mathrm{ml}$ of $0.75 \%$ ropivacaine) using computer generated random number list. Group allocation was concealed in sealed and opaque envelopes. Inclusion criteria consisted of patients belonging to ASA 1 or 2 , in the age group 18-75 years, weighing $40-85 \mathrm{kgs}$, who were planned electively for ACL reconstruction under spinal anesthesia and who gave informed consent. Patients with ASA $\geq 3$, BMI $>35$, patients with impaired liver or kidney function, history of alcohol or drug abuse, history of chronic pain condition or daily intake of analgesics and steroids, uncontrolled anxiety, schizophrenia or bipolar disorder, with hypersensitivity to amide local anaesthetics, peripheral neuropathy, were excluded from the study.

\section{Procedure}

\section{One Day before Surgery}

Details of present study process including the sequence of anesthetic procedures and potential side effects were explained to all patients and relatives and informed consent was taken. The patients were also informed about the Numerical Rating Scale (NRS) for assessment of pain postoperatively. Thorough clinical examination \& history were taken. Routine investigations such as CBC (complete blood count), ECG and x-ray Chest of patients, Blood sugar, RFT (Renal function tests), coagulation profile, blood group, 2D-ECHO (for required patients) were done after consultation with physician. Patients were kept NBM (Nil by mouth) for 6 hours and oral sedative premedication was given night before surgery.

\section{Intraoperative Procedure}

Patients were co-loaded with $10-15 \mathrm{ml} / \mathrm{kg}$ Ringers Lactate. All standard monitors (NIBP, ECG, pulse oximeter) were attached. Patient's baseline systolic/diastolic/mean BP, heart rate, $\mathrm{SpO} 2$ along with respiratory rate were noted. Equipments and drugs necessary for resuscitation and general anaesthesia administration were kept ready. With the patient in lateral decubitus position using midline approach, under all aseptic precautions, skin infiltration was done with $2 \mathrm{ml}$ of $2 \%$ lignocaine in L2-3 or L3-4 interspaces in lateral decubitus position and then subarachnoid block was achieved using 25 G Quinke's spinal needle with $3 \mathrm{ml}$ of $0.5 \%$ bupivacaine (heavy).

All patients were monitored during the course of anaesthesia \& surgery with a common protocol. Time of start of surgery (from skin incision) and total duration of surgery was noted among all the patients. Hypotension was managed by giving injection mephentermine 3-6mg i.v. boluses; bradycardia was managed with inj.Atropine $0.6 \mathrm{mg}$ i.v. boluses.

\section{Technique of USG Guided ACB Block}

An ultrasound (Ultrasound machine with linear, high frequency $(7-13 \mathrm{MHz})$ probe (sonosite version) guided adductor canal block (ACB) block was performed in postoperative ward. The ACB block was performed in a supine position with the extremity to blocked externally rotated. On junction between upper $2 / 3$ rd and lower $1 / 3$ rd of thigh, at the mid-point between the inguinal crease and medial condyle, a $10-12 \mathrm{MHz}$ transducer probe placed in transverse orientation to visualize the femoral artery in short axis deep to Sartorius muscle. Under aseptic precautions, the skin was infiltrated with $2-3 \mathrm{ml}$ of $2 \%$ lignocaine with a $26 \mathrm{~g}$ $\mathrm{h} 1 / 2$ inch hypodermic needle. The block was performed in plane to the ultrasound beam with 21 gauge, $100 \mathrm{~mm}$, short bevel needle (stimuplex, B -Braun) to position needle tip anterolateral to the artery and just deep to posterior fascia of Sartorius muscles. When necessary, small aliquots of D5W were used for hydrolocalisation of needle tip. Once in position a total volume of $20 \mathrm{ml}$ of $0.75 \%$ ropivacaine $1.5 \mathrm{mg} / \mathrm{kg}$ (upto a maximum of $150 \mathrm{mg}$ ) was deposited adjacent to femoral artery and deep Sartorius muscle, with intermittent aspiration. Ropivacaine provides greater sensorimotor differential block, has shorter elimination half time with lower potential for accumulation, lesser cardiovascular toxicity and increased margin of safety over bupivocaine. ${ }^{14}$ After completion of procedure, a sterile dressing was placed over insertion site.

At the end of surgery, as part of hospital protocol, multimodal analgesic regimen consisting of intravenous paracetamol $1 \mathrm{gm} \&$ injection diclofenac $75 \mathrm{mg}$ were given on admission to PACU (Post-operative Care Unit) in both the groups. Additionally, for the ACB group, USG guided ACB was performed by the anaesthesiologist with $20 \mathrm{ml} 0.75 \%$ ropivacaine. 
The postoperative pain was assessed at rest \& on movement using 0-10 numerical rating scale (NRS), also known as the Numerical Pain Intensity Scale (NPIS). ${ }^{13}$ In this scale, patients rated their pain on a 0 -to-10 scale where 0 represented 'no pain at all', rating of 1-3 represented mild, 46 represented moderate and 7-10 represented severe pain. Pain intensity levels were measured post-op upto 24 hours. Static pain was the pain when patient was in bed rest and dynamic pain was when patient was ambulated. The NRS scores were assessed immediately postop, at $30 \mathrm{~min}, 1$ hour, $2,4,8,12,16,20$ and 24 hours postop, by a pain nurse who did not know the details of the groups to which the patients were allocated and was trained enough to take the relevant readings of pain score.

Whenever the patient's NRS $>3$, intravenous tramadol $50-100 \mathrm{mg}$ boluses (upto a maximum of $400 \mathrm{mg}$ ) along with antiemetic injection ondansetron $0.1 \mathrm{mg} / \mathrm{kg}$ were given to both the study groups.

\section{Outcome Measures}

Postoperatively patients were assessed by a) Time of first request for tramadol after completion of surgery in both groups. b) Total dose of tramadol received and frequency in the first $24 \mathrm{hrs}$ postoperatively in both groups. c) Static and dynamic NRS pain scores in $24 \mathrm{hrs}$ postoperatively.

\section{Results}

A total of 60 patients completed the study, 30 in the conventional group and 30 in the ACB group. Data Analysis was done with the Statistical software Windostat version 9.2 and Microsoft word and Excel have been used to generate tables and graphs. Student's unpaired t-test, Chi-square test / Fisher Exact test, were applied to estimate the difference between two groups. Tests with $\mathrm{p}$ value $<0.05$ were considered statistically significant.

The two groups of patients were comparable in age, height and weight. The average age in conventional group was 27.5+6.6yrs and average age in ACB group was $26.6+7.1 \mathrm{yrs}$. The average weight in conventional group was $58.2 \pm 5.7 \mathrm{Kgs}$ and in ACB Group was $59.6 \pm 3.9 \mathrm{Kgs}$. The average height in conventional group was $157.6 \pm 4.4 \mathrm{~cm}$ and in ACB Group was $158.3 \pm 4.4 \mathrm{~cm}$. There was no statistically significant difference in age, weight and height among the two study groups. (Table 1)

The ASA physical status grade in conventional group was $1.03 \pm 0.18$ and in ACB Group was $1.07 \pm 0.25$. The average duration of surgery in conventional group was 98.0 \pm 3.87 mins and in ACB group was $94.83 \pm 4.42$ mins. There was no statistically significant difference in The ASA physical status grade and the duration of surgery between the two study groups. (Table 1)

The mean static pain score on Numerical Rating Scale in conventional group and ACB group was 1.0 respectively at POSTOP after surgery (Table 2). The difference in the two groups was not statistically significant ( $\mathrm{p}$ value $=1.00$ ). Patients in the conventional group reported significantly higher NRS static pain scores at 1 hour $(1.6 \pm 0.65)$, at 2 hours $(1.5 \pm 0.6)$, at 4 hours $(1.9 \pm 0.6)$, at 8 hours $(2.0 \pm 0.5)$, at 12 hours $1.9 \pm 0.6$, at 20 hours $(2.06 \pm 0.449)$ and 24 hours $(2.00$ \pm 0.0 ), when compared with the patients of ACB group at 1 hour $(1.0 \pm 0.00)$, at 2 hours $(1.0 \pm 0.0)$, at 4 hours $(1.2 \pm 0.6)$, at 8 hours $(1.4 \pm 0.67)$, at 12 hours $(1.40 \pm 0.66)$, at 20 hours $(1.77 \pm 0.50)$ and at 24 hours $(1.53 \pm 0.5)$. This difference in the static NRS scores at these hours was found to be statistically significant, except for the $16^{\text {th }}$ hour static NRS score

Similarly, there was statistically significant reduction in the mean dynamic pain score on Numerical Rating Scale among patients in the ACB group when compared with the conventional group from $1^{\text {st }}$ hour upto 24 hours except at the $16^{\text {th }}$ hour postop. (Table 3 )

The mean time of first request of tramadol in the conventional group was $1.717 \pm 0.18 \mathrm{hrs}$ and that in the ACB group was $15.33 \pm 0.745 \mathrm{hrs}$ and this difference was found to be statistically significant. The patient's tramadol dose frequency in the conventional group and ACB group were $3.60+0.67$ and $1.2+0.45$ respectively and this difference was statistically significant. The postoperative $24 \mathrm{hr}$ total tramadol consumption in conventional group $(333.3 \pm 12.76 \mathrm{mg})$ was significantly higher than the consumption in the ACB group $(115.0 \pm 7.638 \mathrm{mg})$ [Table 4]

Table 1: Patient's characteristics

\begin{tabular}{|c|c|c|c|c|c|c|}
\hline \multirow{2}{*}{ Study parameter } & \multicolumn{2}{|c|}{$\begin{array}{c}\text { Conventional tramadol } \\
\text { Group (n=30) }\end{array}$} & \multicolumn{2}{|c|}{ ACL Group (n=30) } & \multicolumn{2}{c|}{ 't' test value } \\
\cline { 2 - 6 } & Mean & Std.Dev. & Mean & Std.Dev. & & \\
\hline Age (years) & 27.50 & 6.6 & 26.60 & 7.1 & 0.5 & 0.6 \\
\hline Height(cm) & 157.6 & 4.4 & 158.3 & 4.4 & 0.613 & 0.5 \\
\hline Weight(kg) & 58.2 & 5.7 & 59.60 & 3.97 & 1.1 & 0.3 \\
\hline ASA grade I & $27(90 \%)$ & & $28(93.3 \%)$ & & & 0.561 \\
\hline ASA grade II & $3(100.0 \%)$ & & $2(6.7 \%)$ & & Chi sq: 0.218 & 0.640 \\
\hline Duration of surgery & 98.0 & 3.877 & 94.83 & 4.424 & 0.538 & 0.592 \\
\hline
\end{tabular}


Table 2: Comparison of postoperative static NRS (Numerical Rating Scale) pain scores in the two groups

\begin{tabular}{|c|c|c|c|c|c|c|}
\hline \multirow{2}{*}{$\begin{array}{c}\text { Static Pain NRS scores } \\
\text { postoperatively }\end{array}$} & \multicolumn{2}{|c|}{$\begin{array}{c}\text { Conventional tramadol } \\
\text { Group }(\mathbf{n}=\mathbf{3 0})\end{array}$} & \multicolumn{2}{|c|}{ ACL Group (n=30) } & \multirow{2}{*}{ t test } & \multirow{2}{*}{ 'p' Value } \\
\cline { 2 - 5 } & Mean & Std.Dev & Mean & Std.Dev & & \\
\hline Immediately Post operation & 1.00 & - & 1.00 & - & - & 1.000 \\
\hline $30 \mathrm{~min}$ & 1.066 & 0.365 & 1.00 & 0.00 & 1.000 & 0.321 \\
\hline $1 \mathrm{hr}$ & 1.633 & 0.6149 & 1.00 & 0.00 & 5.641 & 0.000 \\
\hline $2 \mathrm{hr}$ & 1.4667 & 0.5713 & 1.00 & 0.00 & 4.474 & 0.000 \\
\hline $4 \mathrm{hr}$ & 1.933 & 0.7849 & 1.1667 & 0.379 & 4.818 & 0.000 \\
\hline $8 \mathrm{hr}$ & 2.00 & 0.4549 & 1.3667 & 0.6687 & 4.289 & 0.000 \\
\hline $12 \mathrm{hr}$ & 1.933 & 0.5833 & 1.433 & 0.6261 & 3.201 & 0.002 \\
\hline $16 \mathrm{hr}$ & 2.1667 & 0.4611 & 2.20 & 0.9248 & 0.177 & 0.860 \\
\hline $20 \mathrm{hr}$ & 2.0667 & 0.4498 & 1.7667 & 0.504 & 2.432 & 0.018 \\
\hline $24 \mathrm{hr}$ & 2.00 & 0.000 & 1.533 & 0.5074 & 5.037 & 0.000 \\
\hline
\end{tabular}

Table 3: Comparison of postoperative dynamic NRS scores in the two groups

\begin{tabular}{|c|c|c|c|c|c|c|}
\hline $\begin{array}{c}\text { Study } \\
\text { Parameter } \\
\text { Dynamic NRS }\end{array}$ & \multicolumn{2}{|c|}{$\begin{array}{c}\text { Conventional tramadol } \\
\text { Group }(\mathbf{n}=\mathbf{3 0})\end{array}$} & \multicolumn{2}{c|}{ ACL Group (n=30) } & t test & 'p' Value \\
\cline { 2 - 5 } & Mean & Std.Dev. & Mean & Std.Dev. & & \\
\hline Postop & 1.000 & 0.000 & 1.000 & 0.000 & & 1.000 \\
\hline $30 \mathrm{~min}$ & 1.266 & 0.784 & 1.033 & 0.182 & 1.586 & 0.118 \\
\hline $1 \mathrm{hr}$ & 3.133 & 1.852 & 1.133 & 0.345 & 5.814 & 0.000 \\
\hline $2 \mathrm{hr}$ & 3.166 & 1.577 & 1.233 & 0.430 & 6.476 & 0.000 \\
\hline $4 \mathrm{hr}$ & 3.700 & 1.764 & 1.66 & 0.660 & 5.910 & 0.000 \\
\hline $8 \mathrm{hr}$ & 3.900 & 1.538 & 2.200 & 1.156 & 4.836 & 0.000 \\
\hline $12 \mathrm{hr}$ & 2.933 & 1.201 & 2.400 & 1.191 & 1.726 & 0.090 \\
\hline $16 \mathrm{hr}$ & 3.800 & 1.517 & 4.066 & 1.617 & 0.659 & 0.513 \\
\hline $20 \mathrm{hr}$ & 4.200 & 1.471 & 3.100 & 1.4227 & 2.944 & 0.005 \\
\hline $24 \mathrm{hr}$ & 2.100 & 0.402 & 2.166 & 0.4611 & 0.597 & 0.553 \\
\hline
\end{tabular}

Table 4: Time of first request for tramadol by participants

\begin{tabular}{|l|c|c|c|c|c|c|}
\hline \multicolumn{1}{|c|}{ Study Parameter } & \multicolumn{2}{|c|}{$\begin{array}{c}\text { Conventional tramadol group } \\
(\mathbf{n = 3 0})\end{array}$} & \multicolumn{2}{c|}{ ACB Group (n=30) } & t test & 'p' Value \\
\cline { 2 - 5 } & Mean & Std.Dev & Mean & Std.Dev & & \\
\hline $\begin{array}{l}\text { Mean time to first request } \\
\text { of analgesia (in hours) }\end{array}$ & 1.717 & 0.188 & 15.333 & 0.745 & 17.724 & 0.0000 \\
\hline Dose frequency of tramadol & 3.600 & 0.675 & 1.267 & 0.450 & 15.762 & 0.000 \\
\hline 24hr tramadol (mg) & 333.3 & 12.76 & 115.0 & 7.638 & 14.681 & 0.00 \\
\hline
\end{tabular}

\section{Discussion}

"Pain is what patient says, hurts". Thus the emphasis is on patient's experience. Pain is not just a physical sensation but also an emotional experience. Pain is derived from Latin word 'Poena"' means punishment. It has been described in terms of danger very aptly by the International Association for the Study of Pain (IASP) as "An unpleasant sensory and emotional experience associated with actual or potential tissue damage or described in terms of such damage". ${ }^{15}$

The appropriateness of postoperative analgesic technique after arthroscopic ACL reconstruction surgery is always debatable. Different techniques have been described to provide postoperative analgesia such as epidural analgesia, sub-arachnoid blockade, local wound infiltration and selective femoral nerve blockade. The choice of technique might vary depending upon doctors' and patients' preferences, co-morbidities and type of surgery. ${ }^{2-6}$ Every method has its own advantages and limitations. In general, local or wound infiltration is safe but limited in terms of duration of action, depending on the pharmacodynamics of the local anaesthetic agent used. ${ }^{6}$ Spinal and epidural are safe but limited in term of pharmacodynamics of local anaesthetic agent used and the motor blockade which delay the patient recovery and also physiotherapy.

A reconstruction surgery is associated with moderate to severe postoperative pain which can be managed by peripheral nerve block. With recent application of ultrasound in anaesthetic practice, several techniques of peripheral nerve block like femoral nerve block and adductor nerve block are performed. Even though femoral nerve block provides superior analgesia when compared to traditional intraarticular injection of local anaesthesia, its use is limited in less invasive ambulatory knee surgeries because it results in motor blockade of the quadriceps with potential risk of falling. ${ }^{1,5-7}$ Adductor canal block is almost exclusively 
sensory block that has been demonstrated to reduce the pain $\&$ opioid consumption after knee surgery. ${ }^{8}$

In the present study, 60 ASA physical status I \& II patients scheduled for elective ARTHROSCOPIC anterior cruciate ligament (ACL) repair were randomly allocated into conventional analgesia group $(n=30)$ and ACB group $(n=30)$, to assess the postoperative analgesic efficacy among them. Rescue analgesics in the form of tramadol (maximum $400 \mathrm{mg}$ ) were given to the patients of both the study groups who complained of pain with NRS score $>3$.

In our study, the NRS static and dynamic Scores among the patients of ACB group were significantly lower than the NRS static and dynamic scores among the patients of conventional group at $1^{\text {st }}, 2^{\text {nd }}, 4^{\text {th }}, 8^{\text {th }}, 12^{\text {th }}, 20^{\text {th }}$ and $24^{\text {th }}$ hour postop, whereas the pain scores were not significant at postop (0 mins), 30min and at $16^{\text {th }} \mathrm{hr}$. (Table 2 and 3 ).similar results have been found in studies conducted by Jin SQ et al, ${ }^{16}$ Jenstrup MT et a ${ }^{17}$ and Grevstad $\mathrm{U}$ et al ${ }^{18}$ where the authors have documented that the adductor canal block resulted in significantly lesser dynamic and static pain scores among the patients undergoing total knee arthroplasty.

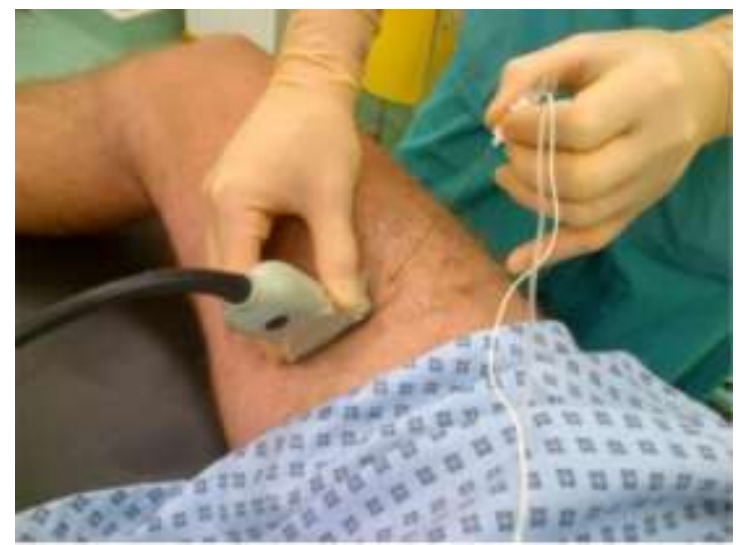

Fig. 1: Patient positioning in adductor canal approach

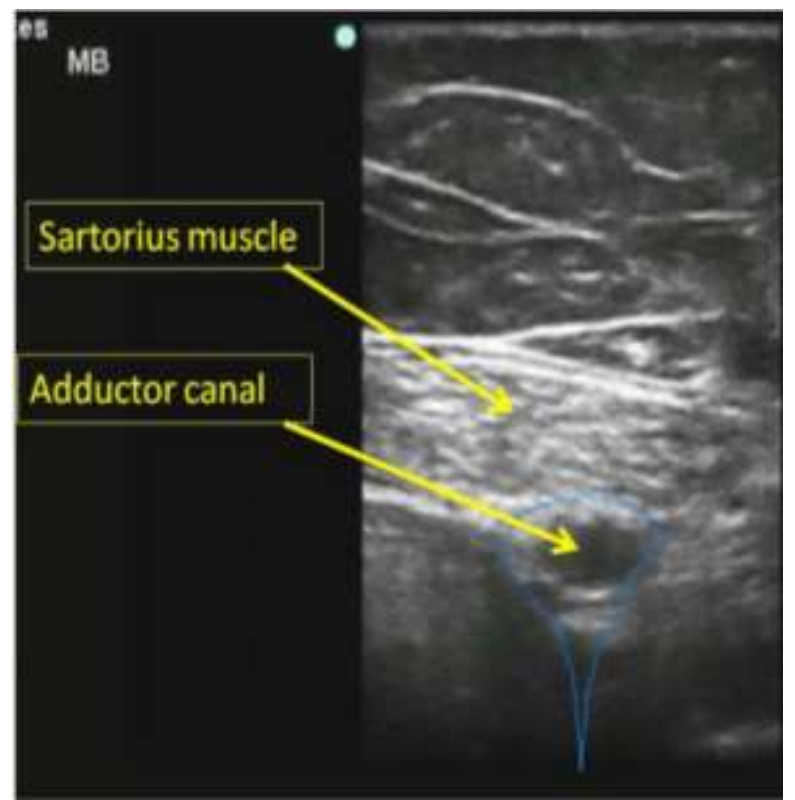

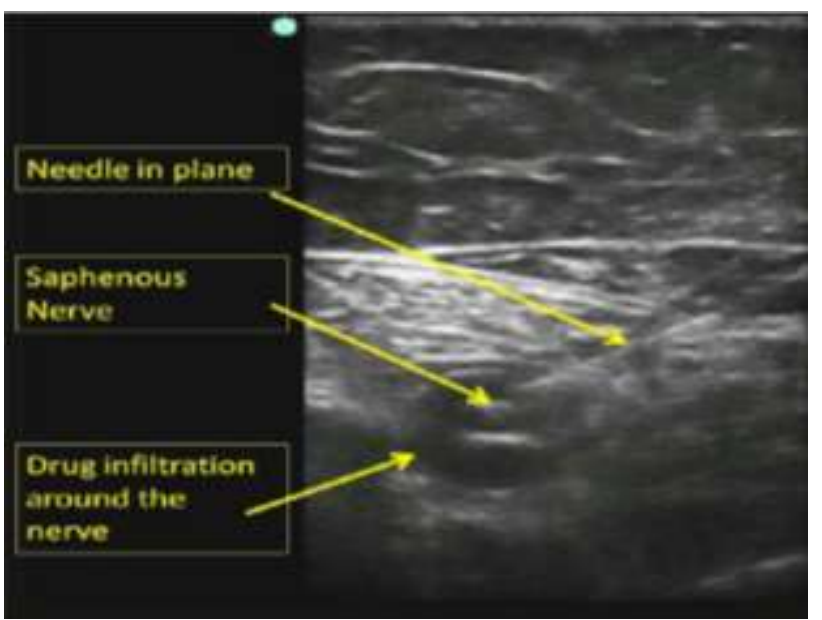

Fig. 3: Needle traversing Sartorius and tip within adductor canal (in-plane needle approach) and local anaesthetic spreading under Sartorius within adductor canal

\section{First Request for Rescue Analgesia}

It is found that in ACB group rescue analgesia was required at a later stage postop when compared to conventional analgesia group among whom $100 \%$ rescue analgesia was required. The first request for analgesia was significantly earlier in conventional group $(1.717 \pm 0.19$ hours) when compared with the ACB group $(15.33 \pm 0.75$ hours). Similar results were seen in study conducted by Jin SQ et $\mathrm{al}^{16}$ where saphenous nerve block resulted in significantly less morphine consumption during the first 24 postoperative hours.

\section{Frequency and total Dosage of Tramadol Required}

In our study, dose frequency of tramadol among patients in conventional group (3.60+0.674) was significantly higher than that among the patients in ACB group (1.2+0.45). Also, the total analgesic dose requirement for the first $24 \mathrm{hrs}$ in conventional group $(333.3 \pm 12.76 \mathrm{mg}$ of tramadol) was significantly higher than that required among the patients of ACB group (115.0 $\pm 7.638 \mathrm{mg}$ ). Our study results are comparable with the studies conducted by Jenstrup MT et $\mathrm{al},{ }^{17}$ and Hanson NA et al, ${ }^{19}$ where it is documented that morphine consumption within first 24 hours was significantly reduced in the ACB group compared to the placebo group.

\section{Conclusion}

Thus this randomized controlled trial demonstrates that USG guided ACB block, as a component of multimodal analgesic regimen provides superior analgesia when compared to conventional analgesia during the $24 \mathrm{hr}$ postoperative period among patients undergoing elective arthroscopic ACL repair. The Adductor canal block reduced postoperative tramadol consumption, increased time to first request of tramadol, decreased NRS Scores at rest and movement.

Fig. 2: The adductor canal beneath Sartorius 


\section{Limitations of the Study}

In the present study, the observation was done only for the first $24 \mathrm{hrs}$ postoperatively and not 48 hours. There are few areas of uncertainty like optimal dosing schemes (i.e., single shot versus catheter, intermittent versus continuous catheter infusions, type of local anaesthetics, use of adjuvants), coagulation status which require further research.

\section{Financial Support and Sponsorship: Nil.}

\section{Conflicts of Interest: None.}

\section{References}

1. Iskandar H, Benard A, Ruel-Raymond J. Femoral block provide superior analgesia compared with intraarticular ropivacaine after anterior cruciate ligament reconstruction. Reg Anesth Pain Med 2003;28(1):29-32.

2. Ready LB. American Society of Regional Anesthesia 1999 Gaston Labat Lecture-Acute pain: Lessons learned from 25,000 patients. Reg Anesth Pain Med 1999;24(6):499-505.

3. Hermanides J, Hollmann MW, Stevens MF, Lirk P. Failed epidural: causes and management. Br J Anaesth 2012;109(2):144-54.

4. Fowler SJ, Symons J, Sabato S, Myles PS. Epidural analgesia compared with peripheral nerve blockade after major knee surgery: a systematic review and meta-analysis of randomized trials. Br J Anaesth 2008;100(2):154-64.

5. Ilfeld BM, Duke KB, Donohue MC. The association between lower extremity continuous peripheral nerve blocks and patient falls after knee and hip arthroplasty. Anesth Analg 2010;111(6):1552-4.

6. Muraskin SI, Conrad B, Zheng N, Morey TE, Enneking FK. Falls associated with lower-extremity-nerve blocks: a pilot investigation of mechanisms. Reg Anesth Pain Med 2007;32(1):67-72.

7. Sung DH, Jung JY, Kim HD, Ha BJ, Ko YJ. Motor branch of the rectus femoris: anatomic location for selective motor branch block in stiff-legged gait. Arch Phys Med Rehabil 2003;84(7):1028-31.

8. Espelund M, Fomsgaard JS, Haraszuk J, Mathiesen O, Dahl JB. Analgesic efficacy of ultrasound-guided adductor canal blockade after arthroscopic anterior cruciate ligament reconstruction: a randomised controlled trial. Eur J Anaesth (EJA) 2013;30(7):422-8.

9. Benzon HT, Sharma S, Calimaran A. Comparison of the different approaches to saphenous nerve block. Anesth 2005;102(3):633-8.
10. Ishiguro S, Yokochi A, Yoshioka K, Asano N, Deguchi A, Iwasaki $\mathrm{Y}$ et al. Technical communication: anatomy and clinical implications of ultrasound-guided selective femoral nerve block. Anesth Analgesia 2012;115(6):1467-70.

11. Lund J, Jenstrup MT, Jaeger P, Sorensen AM, Dahl JB. Continuous adductor-canal- blockade for adjuvant postoperative analgesia after major knee surgery: preliminary results. Acta Anaesthesiologica Scand 2011;55(1):14-9.

12. Mansour NY. Sub-sartorial saphenous nerve block with the aid of nerve stimulator. Reg Anesth 1993;18(4):266-8.

13. Hjermstad MJ, Fayers PM, Haugen DF, Caraceni A, Hanks GW, Loge JH et al. European Palliative Care Research Collaborative (EPCRC). Studies comparing Numerical Rating Scales, Verbal Rating Scales, and Visual Analogue Scales for assessment of pain intensity in adults: a systematic literature review. J Pain Symptom Manag 2011;41(6):1073-93.

14. Hansen TG. Ropivacaine: A pharmacological review. Expert Rev Neurother 2004;4:781-91.

15. Merskey $\mathrm{H}$, editor. Classification of chronic pain: descriptions of chronic pain syndromes and definitions of pain terms. Pain 1986;Suppl3:226.

16. Jin SQ, Ding XB, Tong Y, Ren H, Chen ZX, Wang X, et al. Effect of saphenous nerve block for postoperative pain on knee surgery: a meta-analysis. Int J Clin Exp Med 2015;8(1):368.

17. Jenstrup MT, Jaeger P, Lund J, Fomsgaard JS, Bache S, Mathiesen O, et al. Effects of adductor-canal blockade on pain and ambulation after total knee arthroplasty: a randomized study. Acta Anaesthesiologica Scand 2012;56:357-64.

18. Jaeger P, Grevstad U, Henningsen MH, Gottschau B, Mathiesen O, Dahl JB. Effect of adductor-canal-blockade on established, severe post-operative pain after total knee arthroplasty: a randomised study. Acta Anaesthesiologica Scand 2012;56(8):1013-9.

19. Hanson NA, Derby RE, Auyong DB, Salinas FV, Delucca C, Nagy R, et al. Ultrasound-guided adductor canal block for arthroscopic medial meniscectomy:a randomized, double-blind trial. Can J Anesth 2013;60(9):874-80.

How to cite this article: Ghodageri S, Rammohan G, Narasannavar A, Fultambkar G, Rashmi BM. A randomized controlled trial to assess the postoperative analgesic efficacy of USG guided adductor canal block compared to conventional analgesia among the patients undergoing arthroscopic anterior cruciate ligament reconstruction. Indian J Clin Anaesth 2019;6(2):229-34. 